\title{
On Concurrently Secure Computation in the Multiple Ideal Query Model
}

\author{
Vipul Goyal $^{1}$ and Abhishek Jain ${ }^{2, \star}$ \\ 1 Microsoft Research, India \\ vipul@microsoft.com \\ 2 MIT and Boston University \\ abhishek@csail.mit.edu
}

\begin{abstract}
The multiple ideal query (MIQ) model was introduced by Goyal, Jain and Ostrovsky [Crypto'10] as a relaxed notion of security which allows one to construct concurrently secure protocols in the plain model. The main question relevant to the MIQ model is how many queries must we allow to the ideal world adversary? The importance of the above question stems from the fact that if the answer is positive, then it would enable meaningful security guarantees in many application scenarios, as well as, lead to resolution of long standing open questions such as fully concurrent password based key exchange in the plain model.

In this work, we continue the study of the MIQ model and prove severe lower bounds on the number of ideal queries per session. Following are our main results:
\end{abstract}

1. There exists a two-party functionality that cannot be securely realized in the MIQ model with only a constant number of ideal queries per session.

2. There exists a two-party functionality that cannot be securely realized in the MIQ model by any constant round protocol, with any polynomial number of ideal queries per session.

Both of these results are unconditional and even rule out protocols proven secure using a non-black-box simulator. We in fact prove a more general theorem which allows for trade-off between round complexity and the number of ideal queries per session. We obtain our negative results in the following two steps:

1. We first prove our results with respect to black-box simulation, i.e., we only rule out simulators that make black-box use of the adversary.

2. Next, we give a technique to "compile" our negative results w.r.t. black-box simulation into full impossibility results (ruling out nonblack-box simulation as well) in the MIQ model. Interestingly, our compiler uses ideas from the work on obfuscation using tamper-proof hardware, even though our setting does not involve any hardware.

\footnotetext{
* This material is based on research sponsored by NSF grant \#1218461 and DARPA under agreement number FA8750-11-2-0225. The U.S. Government is authorized to reproduce and distribute reprints for Governmental purposes notwithstanding any copyright notation thereon. The views and conclusions contained herein are those of the authors and should not be interpreted as necessarily representing the official policies or endorsements, either expressed or implied, of DARPA or the U.S. Government. The author also thanks RISCS (Reliable Information Systems and Cyber Security) institute.
} 


\section{Introduction}

The notion of secure computation is central to cryptography. Introduced in the seminal works of [4318], secure multi-party computation allows a group of (mutually) distrustful parties $P_{1}, \ldots, P_{n}$, with private inputs $x_{1}, \ldots, x_{n}$, to jointly compute any functionality $f$ in such a manner that the honest parties obtain correct outputs and no group of malicious parties learn anything beyond their inputs and prescribed outputs.

The classical results for secure computation are only in the stand-alone setting where security holds only if a single protocol session is executed in isolation. Unfortunately, as it has become increasingly evident over the last two decades, stand-alone security does not suffice in real-world scenarios where several protocol sessions may be executed concurrently - a typical example being protocols executed over modern networked environments such as the Internet.

Background: Concurrently Secure Computation. Towards that end, the last decade has a seen a significant effort by the cryptographic community towards obtaining protocols that are concurrently composable, i.e., protocols that remain secure even when executed concurrently over an insecure network. For example, we could require security under concurrent self-composition (which is the focus of this work): a protocol should remain secure even when there are multiple copies executing concurrently. The framework of universal composability (UC) [7] was introduced to capture the setting of concurrent general composition, where a protocol may be executed concurrently not only with several copies of itself but also with other arbitrary protocols.

General positive results for UC secure computation are known based on various trusted setup assumptions, such as a common random string

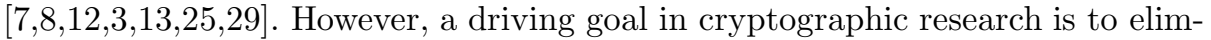
inate the need to trust other entities. As such, positive results for concurrentlysecure computation in the plain model (which is the main focus of this work) are highly desirable, both from a theoretical and practical viewpoint.

Negative Results for Concurrent Composition. Unfortunately, in the plain model, by and large, most of the results have been negative. UC secure protocols for most functionalities of interest were ruled out in 8,10,40,27]. These impossibility results were extended to the setting of general composition by Lindell [30]. Later, Lindell [31] established broad negative results even for the setting of concurrent self-composition by showing equivalence of concurrent self-composition and general composition for functionalities that allow each party to "communicate" to the other via the output. Following the work of Barak et al. [5] and Goyal [20, recently Agrawal et al. [1] and Garg et al. [16] ruled out essentially all non-trivial two-party functionalities for concurrent self-composition, even in the setting where the inputs of honest parties are fixed in advance for all the protocol sessions.

On the positive side, it is known how to realize zero-knowledge and related functionalities, with security in similar models (e.g., [1442 28 395132]). The recent work of Goyal [20] obtains positive results for a broader class of 
functionalities; however, (in keeping with the negative results mentioned above) these results are only relevant to the restricted setting where an honest party uses the same, static input in all of the sessions.

The Search for Relaxed Security Notions. While the above discussion paints a rather bleak picture of state of the art on concurrent security, fortunately, there is a brighter side. Indeed, several prior works have studied relaxations of the standard definition of secure computation that bypass the above negative results, yet provide strong and meaningful security guarantees in the concurrent setting. A well studied notion is that of security w.r.t. super-

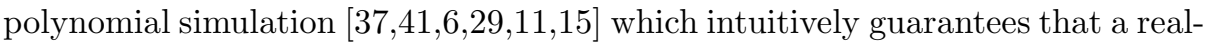
world adversary does not learn any more information than what can be computed in super-polynomial time in the ideal world. Another notion is that of input-indistinguishable computation 3415] which intuitively guarantees that an adversary cannot decide which input (out of possibly many inputs leading to the same output) is used by the honest party in the protocol.

Recently, Goyal, Jain and Ostrovsky [23. introduced the multiple ideal query (MIQ) model for concurrent self-composition where the ideal world adversary is allowed to make more than one output query per session to the ideal functionality. The exact number of queries allowed is a priori fixed by a parameter $\lambda$. In our view, the main advantage of this notion over the previously discussed notions is that it provides an (arguably) intuitive, easy to understand, security guarantee. In particular, in this model, one can precisely measure the amount of "extra" information that the adversary can potentially learn. The security guarantee is provided with standard polynomial time simulation (and adversary) and follows the ideal/real world security formalization. Furthermore, for functionalities such as password-based key exchange, the MIQ definition in fact implies the previous standard definition [19] when $\lambda=\mathrm{O}(1)$.

The MIQ model has also proven relevant in the related setting of resettability. Goyal and Sahai 24] introduced the notion of resettable secure computation and construct such protocols in the model where the ideal adversary can "reset" the trusted party at any point. This gives the ideal adversary the power to query the trusted party multiple times (per session in the real world). This allows them to get a general positive result for all PPT computable functionalities in the plain model in the resettable ideal world setting.

The multiple ideal query model has also proven relevant as technical tool. In particular, the recent positive results of Goyal [20] can be seen as obtained using the following two step paradigm. First, very roughly, Goyal constructs a protocol secure in the multiple ideal query model. Then, the additional queries made to the ideal trusted party are eliminated by constructing an "output predictor".

We believe the study of the MIQ model is well motivated: both because the guarantee provided in the concurrent setting is interesting and non-trivial on its 
own, as well as the connection it has to constructing secure protocols in other related settings.

In this work, we continue the study of the MIQ model.

Our Question: How many Queries? The main question relevant to the MIQ model is how many queries must we allow to the ideal world adversary? Note that if we allow a large number of queries, then the security guarantee may quickly degrade and become meaningless; in particular, the adversary may be able to learn the input of the honest party in the worse case. On the other hand, if the number of allowed queries is very small, say $1+\epsilon$ per session, then the security guarantee is very close to that of the standard definition.

To exemplify this further, consider the oblivious polynomial evaluation functionality 35,36] where two parties wish to jointly evaluate a polynomial over a point. The input of party $P_{1}$ is a polynomial $Q$, while the input of $P_{2}$ is a point $\alpha$. At the end of the protocol, the party $P_{2}$ gets $Q(\alpha)$ as the output. This is a natural functionality with applications to list intersection, mutual authentication, metering on the web, etc (see [36] for more details on these).

Now, note that if we only allow, say, 2 queries to a malicious $P_{2}$ in the ideal world (per real world session), then as long as $Q$ is a high-degree polynomial, the security guarantee for $P_{1}$ is still quite meaningful. Instead of a single point, now a malicious adversary may learn the output on two points of its choice (from an exponential domain of points). The adversary still does not learn any information about what the polynomial evaluates to on rest of the (exponential) domain. On the other hand, if we allow too many queries (exceeding the degree of the polynomial), then the ideal world adversary may be able to learn the entire polynomial $Q$ thus rendering the security guarantee meaningless.

The only known positive results in the MIQ model are due to 2321]. Goyal et. al. 23. provide a construction where the average number of queries in the ideal world per real world session is a constant (with the constant depending upon the adversary). This was further improved in a recent result 21] which provides a construction where the average number of ideal queries in any session are $\left(1+\frac{1}{\operatorname{poly}(n)}\right)$.

If the guarantee on the number of queries per session is only in expectation, this means that in some sessions, the ideal adversary may still be able to make a large number of queries (while keeping the number of queries low in other sessions). Considering the oblivious polynomial evaluation example above, this means that the security in some sessions may be completely compromised. Furthermore, consider the problem of concurrent password based key exchange $26 \quad 17 / 9223$. An interesting question that has remained open so far is designing a concurrent password based key exchange in the plain model where different pair of parties share different (but correlated) passwords. Indeed, this is the most natural setting for password based key exchange (PAKE) and all currently known construction either do not provide concurrent security or are not in the plain model. We note that a positive result in the MIQ model where the number 
of queries per sessions is a strict constant would directly imply a positive result for this problem 1 This raises the following natural question:

"Do there exist concurrent secure protocols in the MIQ model where the number of ideal queries per session is a strict constant?"

\subsection{Our Results}

In this work, we continue the study of the MIQ model and prove severe unconditional lower bounds on the number of ideal queries per session. Following are our main results:

1. There exists a two-party functionality that cannot be securely realized in the MIQ model with only a constant number of ideal queries per session.

2. There exists a two-party functionality that cannot be securely realized in the MIQ model by any constant round protocol, with any polynomial number of ideal queries per session.

Both of these results are unconditional and even rule out protocols proven secure using a non-black-box simulator. We in fact prove a more general theorem that provides a trade-off between the round-complexity and the number of ideal queries per session.

Let $\operatorname{ceil}_{y}(x)$ be recursively defined as $\operatorname{ceil}_{y}(x)=\left\lceil x \cdot \operatorname{ceil}_{y-1}(x)\right\rceil$. Our main result is stated as follows:

Theorem 1. There exists a two-party functionality $f$ such that for any $d=d(k)$ and $n=n(k)$ that satisfy $n^{d}=\operatorname{poly}(k)$, no $n$-round protocol $\Pi$ securely realizes $f$ in the MIQ model with at most $\lambda=\operatorname{ceil}_{d}\left(1+\frac{1}{n}\right)$ number of queries per session.

Application to Concurrent Precise Zero-Knowledge. While our main results concern with the MIQ model, interestingly, they also find applications in the setting of precise simulation [33. Recall that in the setting of precise simulation, we wish to ensure that the resource utilization of the simulator is "close" to the resource utilization of the adversary in the real world interaction. The resource being studied is typically the running time, however, previous works have also considered a more general setting where the resource in question can be, e.g., memory. One can consider a general setting, where instead of focusing only on a particular resource (such as time), we consider many resources at the same time, such as memory, cache, power, etc. A general question we may ask is whether it is possible to perform simulation that achieves precision for each of these resources simultaneously.

To be more concrete, say we have a concurrent adversary interacting with the prover in many sessions and making use of $k$ different resources (each resource

${ }^{1}$ The first positive result for concurrent PAKE in the plain model provided by Goyal et. al. 23. was for the single password setting where there is a single global correct password which every party is required to use for authentication. This restriction stems from their solution requiring a constant number of ideal queries per session on an average. 
may be utilized by the adversary at any arbitrary point). A natural question is: can one obtain a zero-knowledge simulator such that its utilization of each resource is only within a constant factor of the adversary? Our negative results directly imply a negative answer for this question as well where the number of resources is equal to the number of sessions. In other words, viewing the ideal functionality query in session $i$ as a utilization of the $i$-th resource, we directly have that the simulator will end up going over a constant factor for at least one of the resources. Similarly, our results also imply a severe lower bound for constant round protocols: there exists a resource whose utilization will, in fact, not be within any polynomial factor of the adversary's utilization.

Independent of our work, Pass 38 has been able to obtain a positive result in the stand-alone setting for the above problem. In particular, 38 gives a construction where the simulator is able to be precise in multiple resources simultaneously in the standalone setting.

\subsection{Our Techniques}

In this section, we give an overview of our techniques. We obtain our negative results in the following two steps: we first prove our results with respect to black-box simulation, i.e., we only rule out simulators that make black-box use of the adversary. Next, we give a technique to "compile" our negative results w.r.t. black-box simulation into full impossibility results (ruling out non-blackbox simulation as well) in the MIQ model. Below, we discuss each of these steps separately.

Impossibility for Black-box Simulation. Recall that in order to prove security of a two-party computation protocol, we need to demonstrate that for every real world adversary $\mathcal{A}$ that controls one of the parties, there exists an ideal world adversary (or simulator $\mathcal{S}$ ) who can simulate the view of $\mathcal{A}$. Typically, the simulator $\mathcal{S}$ works by extracting the input a used by $\mathcal{A}$ and then querying the ideal functionality with $a$ to receive the correct output; this output is then used to complete the simulation of $\mathcal{A}$ 's view. Now, further recall that the only advantage that a black-box simulator has over the real adversary is the ability to rewind. In other words, a black-box simulator extracts the input of $\mathcal{A}$ by rewinding. However, in the concurrent setting, extracting the input of $\mathcal{A}$ in each session is a non-trivial task. In particular, given an adversarial scheduling, it may happen that in order to extract the input of $\mathcal{A}$ in a given session $s$, the simulator $\mathcal{S}$ rewinds past the beginning of another session $s^{\prime}$ (that is interleaved inside the protocol messages of session $s$ ). When this happens, $\mathcal{A}$ may change its input in session $s^{\prime}$. Thus, the simulator $\mathcal{S}$ would be forced to query the ideal functionality more than once for the session $s^{\prime}$.

We now briefly explain how the above intuition can be further extended to achieve a black-box impossibility result even when the simulator is allowed to make multiple queries to the ideal functionality. For concreteness, let us consider the (simplified) case where the simulator is allowed a fixed constant $C$ number of queries per session. Note that in order to obtain the desired negative result, 
we need to construct a concurrent adversary $\mathcal{A}$ that can force any black-box simulator $\mathcal{S}$ to make more than $C$ queries for at least one session. We now briefly discuss how to construct such an adversary. Let $n$ be the round complexity of the protocol, where $n$ is any polynomial in the security parameter.

Consider the following static adversarial scheduling of messages. Consider an "outer" session (say) s. We will call it a session at level 0 . Now, between every round of messages in the outer session, place a new complete protocol session. We will call these $n$ sessions to be at level 1 . Next, we again place a new complete protocol session between every round of messages in each session at level 1 . Note that this creates $n^{2}$ sessions at level 2. Repeat this process recursively until we reach level $C$, where there are exactly $n^{C}$ sessions. Thus, in total, we have $m=\frac{n^{C+1}-1}{n-1}$ sessions, which is polynomial in the security parameter.

Now, as discussed earlier, a black-box simulator $\mathcal{S}$ must perform at least one rewinding in order to extract the input of $\mathcal{A}$ in the "outer" session $s$. Suppose that $\mathcal{S}$ rewinds the $i^{\text {th }}$ round of session $s$. Then, this immediately implies that the $i^{t h}$ session at level 1 is executed at least twice, which in turn means that $\mathcal{S}$ will be forced to query the ideal functionality twice for that session. Now, note that $\mathcal{S}$ will need to extract $\mathcal{A}$ 's input in each of these two executions in order to complete them successfully. Thus, assuming that (even if) $\mathcal{S}$ rewinds different rounds in each of these two executions, we have that there exist two sessions at level 2 that are each executed three times. Continuing this argument inductively, we can show that for every level $i$, there exists at least one session that is executed $i+1$ times. As a result, we obtain that there exists a session $s^{*}$ at level $C$ that is executed $C+1$ times. If the adversary chooses a different input in each of the $C+1$ executions, we have that $\mathcal{S}$ must query the ideal functionality $C+1$ times for session $s^{*}$. Thus, we conclude that the black-box simulator $\mathcal{S}$, who is only allowed $C$ queries, must fail 2

From Black-Box to Non-Black-Box. We now discuss a compilation technique to transform our negative results for black-box simulation into full impossibility results that rule out non-black-box simulation as well.

Recall that the main advantage that a non-black-box simulator has over a black-box simulator is that the former can make use of the adversary's code. Then, our high-level approach is to "nullify" this advantage by making use of secure program obfuscation. We note, however, that general program obfuscation is known to be impossible [4. Towards this end, our key idea is to use positive results on program obfuscation using stateless tamper-proof hardware tokens by Goyal et. al. 22]. In the obfuscation with hardware model, one can take the given program and convert it into an obfuscated program using an obfuscation key $k$. The obfuscated program, for execution, would require oracle access to a hardware token having the obfuscation key $k$. We denote the functionality of the token (required to run the obfuscated program) by $f_{\text {token }}$ (parameterized by the key $k)$.

\footnotetext{
${ }^{2}$ We remark that in order to prove our general result, a more tight analysis is necessary; see the technical sections.
} 
Very roughly, our idea is to implement the "token functionality" $f_{\text {token }}$ of 22 using two-party computation. An important point is that $f_{\text {token }}$ is "robust" to any polynomial number of queries; thus, it is particularly suited to the MIQ model. In more detail, we obtain our negative result in the following three steps:

Toy Experiment: Let $\Pi$ be any protocol for the $f_{\text {token }}$ functionality and let $\mathcal{A}$ be any concurrent adversary for $\Pi$ that rules out black-box simulators that make at most $\lambda$ queries per session. We first consider a toy experiment involving three parties, namely, Alice, Bob and David. In this experiment, Alice and Bob interact in multiple ideal world executions of the token functionality $f_{\text {token }}$. At the same time, Bob and David are involved in concurrent real-world executions of $\Pi$ where Bob and David follow the same scheduling of messages as defined by adversary $\mathcal{A}$. Furthermore, the adversary Bob is allowed to reset David at any point during their interaction.

David, who has a secret input secret is instructed to reveal secret to Bob if all the executions of $\Pi$ are completed "successfully". The goal of Bob is to successfully complete its interaction with David and learn the value secret. Then, the main idea in this experiment is that, by relying on our black-box impossibility result, we show that no adversarial Bob can succeed in learning secret, except with negligible probability.

Ideal World: In the second step, we eliminate David from the above experiment by obfuscating his next-message function in the $f_{\text {token-hybrid model }}$ and give it as an auxiliary input to Bob (while the corresponding obfuscation "key" is given to Alice). This results in a scenario where Alice and Bob are the only parties, who interact in multiple ideal world executions of $f_{\text {token }}$. From the security of obfuscation, we can argue that this experiment can be reduced to the toy experiment; as such, no adversarial Bob can learn secret, except with negligible probability.

Real World Experiment: We finally consider the real world experiment, which is the same as previous step, except that all the ideal world invocations of $f_{\text {token }}$ are now replaced with real-world executions of protocol $\Pi$. It is not difficult to see that in this experiment, an adversary Bob can simply play a "man-in-the-middle" between Alice and David (since Bob has David's obfuscated code); as a result, Bob can learn secret with probability 1.

From above, it follows that the adversary Bob in the real world experiment is a PPT concurrent adversary for $\Pi$ whose view cannot be simulated by any simulator that makes at most $\lambda$ queries per session, thus yielding us the desired result. We refer the reader to the technical sections for more details.

\section{Preliminaries}

\subsection{Our Model}

In this section, we present our security model. Throughout this paper, we denote the security parameter by $k$. 
Concurrently Secure Computation in the MIQ Model. We define our security model by extending the standard real/ideal paradigm for secure computation. We consider a malicious, static adversary. We do not require fairness and only consider security with abort. Finally, we only consider computational security. We now proceed to describe the ideal and real world experiments and then give our security definition.

IDEAL MODEL. We first define the ideal world experiment, where there is a trusted party for computing the desired two-party functionality $f$. Let there be two parties $P_{1}$ and $P_{2}$ that are involved in multiple sessions, say $m=m(k)$. An adversary may corrupt either of the two parties. As in the standard ideal world experiment for concurrently secure computation, the parties send their inputs to the trusted party and receive the output of $f$ evaluated on their inputs. The main difference from the standard ideal world experiment is that the ideal adversary is allowed to make $\lambda$ (as opposed to one) output queries (with possibly different inputs of its choice) in each session. The ideal world execution proceeds as follows.

Inputs: $P_{1}$ and $P_{2}$ obtain a vector of $m$ inputs, denoted $\boldsymbol{x}$ and $\boldsymbol{y}$ respectively. The adversary is given auxiliary input $z$, and chooses a party to corrupt. Without loss of generality, we assume that the adversary corrupts $P_{2}$ (when the adversary controls $P_{1}$, the roles are simply reversed). The adversary receives the input vector $\boldsymbol{y}$ of the corrupted party.

Session initiation: The adversary initiates a new session by sending a start message to the trusted party. The trusted party then sends (start, $i$ ) to $P_{1}$, where $i$ is the index of the session.

Honest parties send inputs to trusted party: Upon receiving (start, $i$ ) from the trusted party, honest party $P_{1}$ sends $\left(i, x_{i}\right)$ to the trusted party, where $x_{i}$ denotes $P_{1}$ 's input for session $i$.

Adversary sends input to trusted party: Whenever the adversary wishes, it may send a message $\left(i, \ell, y_{i, \ell}^{\prime}\right)$ to the trusted party for any $y_{i, \ell}^{\prime}$ of its choice. Upon sending this pair, it receives back $\left(i, \ell, f\left(x_{i}, y_{i, \ell}^{\prime}\right)\right)$ where $x_{i}$ is the input value that $P_{1}$ previously sent to the trusted party for session $i$. The only limitation is that for any $i$, the trusted party accepts at most $\lambda$ tuples indexed by $i$ from the adversary.

Adversary instructs trusted party to answer honest party: When the adversary sends a message of the type (output, $i, \ell$ ) to the trusted party, the trusted party sends $\left(i, f\left(x_{i}, y_{i, \ell}^{\prime}\right)\right)$ to $P_{1}$, where $x_{i}$ and $y_{i, \ell}^{\prime}$ denote the respective inputs sent by $P_{1}$ and adversary for session $i$.

Outputs: The honest party $P_{1}$ always outputs the values $f\left(x_{i}, y_{i, \ell}^{\prime}\right)$ that it obtained from the trusted party. The adversary may output an arbitrary (probabilistic polynomial-time computable) function of its auxiliary input $z$, input vector $\boldsymbol{y}$ and the outputs obtained from the trusted party.

The ideal execution of a function $f$ with security parameter sec, input vectors $\boldsymbol{x}, \boldsymbol{y}$ and auxiliary input $z$ to $\mathcal{S}$, denoted $\operatorname{IDEAL}_{f, \mathcal{S}}(k, \boldsymbol{x}, \boldsymbol{y}, z)$, is defined as the output pair of the honest party and $\mathcal{S}$ from the above ideal execution. 
Definition 1 ( $\lambda$-Ideal Query Simulator). Let $\mathcal{S}$ be a non-uniform probabilistic (expected) PPT machine representing the ideal-model adversary. We say that $\mathcal{S}$ is a $\lambda$-ideal query simulator if it makes at most $\lambda$ output queries per session in the above ideal experiment.

REAL MODEL. We now consider the real model in which a real two-party protocol is executed (and there exists no trusted third party). Let $f$ be as above and let $\Pi$ be a two-party protocol for computing $f$. Let $\mathcal{A}$ denote a non-uniform probabilistic polynomial-time adversary that controls either $P_{1}$ or $P_{2}$. The parties run concurrent executions of the protocol $\Pi$, where the honest party follows the instructions of $\Pi$ in all executions. The honest party initiates a new session $i$ with input $x_{i}$ whenever it receives a start-session message from $\mathcal{A}$. The scheduling of all messages throughout the executions is controlled by the adversary. That is, the execution proceeds as follows: the adversary sends a message of the form $(i, \mathrm{msg})$ to the honest party. The honest party then adds msg to its view of session $i$ and replies according to the instructions of $\Pi$ and this view. At the conclusion of the protocol, an honest party computes its output as prescribed by the protocol. Without loss of generality, we assume the adversary outputs exactly its entire view of the execution of the protocol.

The real concurrent execution of $\Pi$ with security parameter $k$, input vectors $\boldsymbol{x}$, $\boldsymbol{y}$ and auxiliary input $z$ to $\mathcal{A}$, denoted $\operatorname{REAL}_{\Pi, \mathcal{A}}(k, \boldsymbol{x}, \boldsymbol{y}, z)$, is defined as the output pair of the honest party and $\mathcal{A}$, resulting from the above real-world process.

Definition 2 ( $\lambda$-Secure Concurrent Computation in the MIQ Model). A protocol $\Pi$ is said to $\lambda$-securely realize a functionality $f$ under concurrent self composition in the MIQ model if for every real model non-uniform PPT adversary $\mathcal{A}$, there exists a non-uniform (expected) PPT $\lambda$-ideal query simulator $\mathcal{S}$ such that for all polynomials $m=m(k)$, every pair of input vectors $\boldsymbol{x} \in X^{m}, \boldsymbol{y} \in Y^{m}$, every $z \in\{0,1\}^{*} s,\left\{\operatorname{IDEAL}_{f, \mathcal{S}}(k, \boldsymbol{x}, \boldsymbol{y}, z)\right\}_{k \in \mathbb{N}} \stackrel{c}{=}\left\{\operatorname{REAL}_{\Pi, \mathcal{A}}(k, \boldsymbol{x}, \boldsymbol{y}, z)\right\}_{k \in \mathbb{N}}$.

\subsection{Obfuscation with Tamper-Proof Hardware Tokens}

In this work, we use ideas from the area of obfuscation using tamper-proof hardware tokens. In particular, we use the positive result of Goyal et al. 22] on secure program obfuscation using stateless tamper-proof hardware tokens. Below, we give an abstract overview of the scheme of 22 that we will use in our negative results. We remark that the discussion below hides most of the internal details of the scheme of [22]. We refer the reader to [22] for the details of the scheme.

Obfuscation in Token Hybrid Model. In order to obfuscate a circuit $C$, the sender executes the following steps:

- Sample a token instance $T \leftarrow$ SampleT. The token instance has some secret values hardwired inside it. We will denote the secret values as a key $K$ (that is drawn from some distribution $\mathcal{K}$ ). In other words, sampling of the token instance just involves to sampling the key $K$ from the distribution $\mathcal{K}$.

- Compute the obfuscated program $\mathcal{O}(C) \leftarrow \operatorname{Obfuscate}(C, K)$ 
To compute $C(x)$ on any input $x$, the obfuscated program $\mathcal{O}(C)$ makes $t_{C}$ queries of various types to the token $T$, where each $q$ is drawn from some distribution $\mathcal{Q}$. Here, $t_{C}$, denoted as the query parameter of the obfuscation scheme, is an integer that depends on the size of the circuit $C$ that is obfuscated.

Obfuscating Stateful Programs. The above description is only relevant to obfuscating stateless circuits $C$. We note that it is also possible to obfuscate the programs of stateful (or reactive) machines $M$ in the above scheme by using standard techniques. The basic idea is that obfuscated code $\mathcal{O}(M)$ is computed in such a manner that its output on any given input $x$ consists of both $M(x)$ and an authenticated encryption of its resultant state after the computation of $M(x)$.

We now recall the following security lemma from [22] (informally stated):

Lemma 1 ([22]). Assuming the existence of one-way functions, (SampleT, Obfuscate) is a (stateful) program obfuscation scheme in the token-hybrid model with the following properties. For any adversary having the obfuscated program $\mathcal{O}(C)$ along with oracle access to the token, there exists an ideal world simulator having only black-box access to the circuit $C$, such that, the output distribution of the simulator is computationally indistinguishable from that of the adversary.

The Token Functionality. A key idea that is used in our negative results is to implement the working of the hardware token $T$ via a two-party secure computation protocol. To this end, we abstract the working of the token $T$ as the following two-party functionality $f_{\text {token }}$, that we will refer to as the "token functionality".

Denote by $f_{\text {token }}$ the token functionality with the key $K$ hardwired inside the description of its circuit. The input and output interface of the functionality $f_{\text {token }}$ is described as follows:

Inputs: Party $P_{1}$ gets a token key $K \leftarrow \mathcal{K}$ as input, while $P_{2}$ gets a query $q \leftarrow \mathcal{Q}$ as input.

Outputs: $P_{1}$ gets no output, while $P_{2}$ gets $f_{\text {token }}(K ; q)$.

Note that $f_{\text {token }}$ is a deterministic functionality. We now state a lemma regarding the unpredictability of outputs of $f_{\text {token }}$. We note that this lemma is implicit in $[22]$.

Lemma 2 (Unpredictability of Output of $f_{\text {token }}$ [22]). There exists a distribution $\mathcal{Q}$ (with super-logarithmic min-entropy) from which a query $q$ can be sampled with the following properties. Any adversary $\mathcal{A}$, given oracle access to the functionality $f_{\text {token }}(K ; \cdot)$ (where $K$ is sampled at random from $\mathcal{K}$ ) with the restriction that it is allowed to query $f_{\text {token }}(K ; \cdot)$ on any string except $q$, can output $f_{\text {token }}(K ; q)$ with only negligible probability.

\section{Black-Box Impossibility in the MIQ Model}

In this section, we prove impossibility results for concurrently secure computation in the MIQ model with respect to black-box simulation. Due to lack of 
space, we only state our result statements here and refer the reader to the full version for their formal proofs.

Let $\operatorname{ceil}_{y}(x)$ be recursively defined as $\operatorname{ceil}_{y}(x)=\left\lceil x \cdot \operatorname{ceil}_{y-1}(x)\right\rceil$. Our general result, stated below, shows a trade-off between the query parameter $\lambda$ and the round-complexity $n$ of the protocol:

Theorem 2. There exists a functionality $f$ such that for any $d=d(k)$ and $n=n(k)$ that satisfy $n^{d}=\operatorname{poly}(k)$, no $n$-round protocol $\Pi \lambda=\operatorname{ceil}_{d}\left(1+\frac{1}{n}\right)$ securely realizes $f$ in the MIQ model with respect to black-box simulation.

Note that $\operatorname{ceil}_{d}\left(1+\frac{1}{n}\right) \geq d+1$. Thus, we obtain the following general corollary when substituting $d$ with $\lambda$ :

Corollary 1. There exists a functionality $f$ such that for any $\lambda=\lambda(k)$ and $n=n(k)$ that satisfy $n^{\lambda}=$ poly $(k)$, no $n$-round protocol $\Pi \lambda$-securely realizes $f$ in the MIQ model with respect to black-box simulation.

By plugging in $n=\operatorname{poly}(k)$ and $\lambda=\mathrm{O}(1)$ above, we get the following as a subcorollary, ruling out general positive results in the MIQ model when a (black-box) simulator is allowed only a constant number of ideal queries per session:

Corollary 2. There exists a functionality $f$ that cannot be $\mathrm{O}(1)$-securely realized in the MIQ model with respect to black-box simulation.

Finally, by plugging in $n=O(1)$ and $d=\log (k)$ in Theorem 2, we obtain the following corollary ruling out constant-round protocols in the MIQ model:

Corollary 3. There exists a functionality $f$ that cannot be securely realized in the MIQ model by any $O(1)$-round protocol w.r.t. black-box simulation, even if a (black-box) simulator is allowed any (fixed) poly $(k)$ ideal queries per session.

In fact, we will prove something stronger, as stated below. We first give the following definition:

Definition 3 ( $\lambda$-special black-box adversary). Let $\lambda=\lambda(k)$ and $\Pi=$ $\left(P_{1}, P_{2}\right)$ be a protocol for functionality $f$. A PPT concurrent adversary $\mathcal{A}$ for $\Pi$ that corrupts party $P_{2}$ is said to be $\lambda$-special adversary if the following holds:

- $\mathcal{A}$ outputs accept with probability 1 in the real-world execution with $P_{1}$.

- Except with negligible probability, no $\lambda$-ideal query black-box simulator can send a query to $\mathcal{A}$ such that it outputs accept.

Now, it is easy to see that Theorem 2 is implied by the following theorem:

Theorem 3. For every $d=d(k)$ and $n=n(k)$-round protocol $\Pi$ for the $f_{\text {token }}$ functionality, if $n^{d}=\operatorname{poly}(k)$, then there exists a $\lambda$-special adversary for $\Pi$, where $\lambda=\operatorname{ceil}_{d}\left(1+\frac{1}{n}\right)$. 


\section{Full Impossibility in the MIQ Model}

We now present full impossibility results for concurrently secure computation in the multiple ideal query model, ruling out non-black-box simulation as well. More concretely, we present a technique to "compile" our impossibility results w.r.t. black-box simulation into full impossibility results. We now state our main theorem of this section:

Theorem 4. Let $\lambda=\lambda(k)$ and $\Pi$ be any protocol for the $f_{\text {token }}$ functionality. If there exists a PPT $\lambda$-special black-box adversary $\mathcal{A}$ for $\Pi$, then there exists $a$ PPT concurrent adversary $\mathcal{B}$ for $\Pi$ whose view cannot be simulated by any (potentially non-black-box) PPT $\lambda$-ideal query simulator.

Combining Theorem 3 with the above theorem, we immediately obtain our main result stated in Theorem 10 We also derive the analogous corollaries of Corollary 1, 2, 3, ruling out non-black-box simulation as well. We skip the formal statements here due to lack of space.

\subsection{Proof of Theorem 4}

Let $\lambda=\lambda(k)$ and $\Pi$ be any protocol for the $f_{\text {token }}$ functionality. Then, given any $\lambda$-special black-box adversary $\mathcal{A}$ for $\Pi$, we now show how to construct a $\lambda$-special adversary $\mathcal{B}$ for $\Pi$. We use ideas from obfuscation using stateless tamper-proof hardware tokens [22] in order to show this transformation.

We recommend the reader to review the outline of the three main steps in our proof as described in Section 1.2. We now proceed to describe each of the steps in details. We first setup some notation.

Notation. Let $m$ be the number of sessions that adversary $\mathcal{A}$ schedules for protocol $\Pi$. Let $K_{1}, \ldots, K_{m}$ denote a set of token keys, where each $K_{i}$ is drawn at random from the distribution $\mathcal{K}$. Let $q_{1}, \ldots, q_{m}$ be a set of query strings for the token functionality, where each $q_{i}$ is drawn at random from the distribution $D$ (as defined in Lemma 22). Finally, let secret be a random string in $\{0,1\}^{k}$.

(Toy) Experiment I: Restating the Black-Box Impossibility Result. Consider the following scenario involving three parties, namely, Alice, Bob and David. Alice is given an input vector $\left(K_{1}, \ldots, K_{m}\right)$, while David is given an input vector $\left(\left(K_{1}, q_{1}\right), \ldots,\left(K_{m}, q_{m}\right)\right.$; secret $)$. Here, the input values are chosen in the manner as described above. We describe the interaction between Alice and Bob, and Bob and David, separately.

Interaction between Alice and Bob. Alice and Bob are interacting in $m$ ideal world executions of the functionality $f_{\text {token }}$, where Alice plays the role of $P_{1}$ using input vector $\left(K_{1}, \ldots, K_{m}\right)$ and Bob plays the role of $P_{2}$ using any inputs of its choice. In each of these $m$ ideal world executions, the adversary Bob is allowed to query the token functionality $\lambda$ times using any inputs of its choice.

Interaction between Bob and David. At the same time, Bob and David are interacting in $m$ real-world concurrent executions of protocol $\Pi$, where Bob plays 
the the role of $P_{1}$ with any inputs of its choice and David plays the role of $P_{2}$ by simply running the code of the $\lambda$-special adversary $\mathcal{A}$

Bob and David are instructed to assume the identities of Alice and Bob, respectively, in these sessions. The messages of the $m$ sessions follow the same schedule as defined by the adversary $\mathcal{A}$. Furthermore, the adversary Bob is allowed to reset David at any point during their interaction. That is, at any point during their interaction, Bob can choose to "rewind" David to an earlier state and create new threads of execution.

In each session $i \in[m]$, David verifies whether his output $y_{i}=f_{\text {token }}\left(K_{i}, q_{i}\right)$ (where $q_{i}$ is the input of David in that session, chosen in the same manner as $\mathcal{A}$ would); if this is not the case, then David aborts the interaction with Bob and outputs $\perp$ (i.e., David does not continue any remaining sessions with Bob). If all of the $m$ sessions are completed successfully, then David sends secret to Bob (as the only additional message outside of the $m$ concurrent executions of $\Pi$ ).

Lemma 3. Bob outputs secret in Experiment I with negligible probability.

Experiment II: Ideal World. We now "eliminate" the party David from the above toy experiment. Note that eliminating David results only in interactions between Alice and Bob, which is indeed our desired setting of concurrent selfcomposition. Very roughly, in order to eliminate David, we would like to obfuscate David's next message function and give it as auxiliary input to Bob. Since general program obfuscation is impossible in the plain model [4, our key idea is to use positive results on secure program obfuscation using tamper-proof hardware tokens, and adapt them to our setting. In particular, we will use the positive results of Goyal et al. 222 on secure program obfuscation using a stateless hardware token that implements the $f_{\text {token }}$ functionality. We now give more details. Some of the notation used below is as defined in Section 2.2 .

Eliminating David. Consider the next message function NMF of party David as described in Experiment I. Sample a key $K \leftarrow \mathcal{K}$. Compute the obfuscation

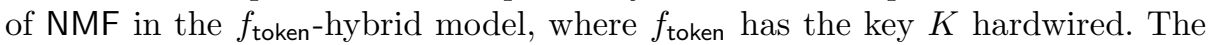
resulting program, denoted as $\mathcal{O}(\mathrm{NMF}) \leftarrow \operatorname{Obfuscate}(K, \mathrm{NMF})$, is given as auxiliary input to Bob. The key $K$, on the other hand, is given as an additional input to Alice.

Ideal World Experiment. Experiment II, or in other words, the ideal world experiment is defined in the same manner as Experiment I, except that we eliminate the party David in the manner as described above. Note that in order to evaluate the program $\mathcal{O}(\mathrm{NMF})$ on any input, Bob would need to answer the queries $q$ of $\mathcal{O}(\mathrm{NMF})$ to the token functionality $f_{\text {token }}(K, \cdot)$. In particular, recall from Section 2.2 that the obfuscated code $\mathcal{O}(C)$ for a program $C$ makes $t_{C}$ queries to the token functionality, where $t_{C}$ (referred to as the query parameter) depends on the size of the circuit $C$. Then, in the ideal world experiment, we have Alice and Bob engage in $t_{\mathrm{NMF}} \cdot m \cdot n$ additional ideal executions of $f_{\text {token }}$, where $t_{\mathrm{NMF}}$ is the

\footnotetext{
${ }^{3}$ In particular, if $\mathcal{A}$ chooses to ignore the inputs $q_{1}, \ldots, q_{m}$ and choose fresh inputs "on-the-fly", then David follows the same strategy.
} 
query parameter for the obfuscation scheme of [22] as determined by the circuit description of NMF, $m$ is the number of sessions that adversary $\mathcal{A}$ schedules, and $n$ is the round complexity of protocol $\Pi$. In each of these $t_{\mathrm{NMF}} \cdot m \cdot n$ executions, Alice uses the key $K$ as input, while Bob is allowed to use any inputs of its choice. Further, in each of these $t_{\mathrm{NMF}} \cdot m \cdot n$ ideal executions, Bob is allowed to query the token functionality $\lambda$ times using any inputs of its choice.

Thus, overall the ideal world experiment between Alice and Bob consists of the following:

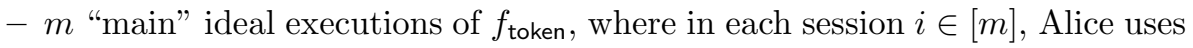
key $K_{i}$ as input, while Bob is allowed to use any inputs of its choice.

$-t_{\mathrm{NMF}} \cdot m \cdot n$ "auxiliary" ideal executions of $f_{\text {token }}$, where in each session, Alice uses key $K$ as input, while Bob is allowed to use any inputs of its choice.

Further, as explained above, in each of the $m+t_{\mathrm{NMF}} \cdot m \cdot n$ ideal executions of $f_{\text {token }}$, Bob is allowed to query the ideal functionality $\lambda$ times using any inputs of its choice.

Lemma 4. Bob outputs secret in Experiment II with negligible probability.

Experiment III: Real World. We now describe the final experiment, which is the real world experiment between Alice and Bob. This experiment is essentially the same as Experiment II, except that each ideal world execution of $f_{\text {token }}$ is now replaced with a real world execution of protocol $\Pi$ between Alice and Bob. In more detail, the interaction between Alice and Bob consists of the following:

- $m$ "main" executions of protocol $\Pi$, where the messages of the $m$ executions are scheduled in the same manner as defined by the adversary $\mathcal{A}$. In each $i \in[m]$ main session, Alice uses the keys $K_{i}$ as her input.

$-t_{\mathrm{NMF}} \cdot m \cdot n$ "auxiliary" executions of protocol $\Pi$ that are scheduled by Bob in the manner, as described below. In each of these auxiliary sessions, Alice uses key $K$ as her input.

Alice's program: Alice is given input the keys $K_{1}, \ldots, K_{n}$ for the $m$ "main" sessions, and key $K$ for the $t_{\mathrm{NMF}} \cdot m \cdot n$ "auxiliary" sessions.

Alice behaves honestly according to the protocol $\Pi$ and responds honestly to all protocol invocations made by Bob by using the code of $P_{1}$.

Bob's program: Bob is given as auxiliary input the obfuscated program $\mathcal{O}(\mathrm{NMF})$, where NMF is the next-message function of David (as described above). Let secret be the secret value hardwired in $\mathcal{O}(\mathrm{NMF})$.

For $i=1, \ldots, m \cdot n$, do:

1. Upon receiving the $i^{\text {th }}$ message from Alice in $m$ main sessions, say $a_{i}$, suspend (temporarily) the ongoing session. Run the code $\mathcal{O}(\mathrm{NMF})$ on input $a_{i}, 4$

${ }^{4}$ Note that the input to $\mathcal{O}(\mathrm{NMF})$ would also include the authenticated state information that $\mathcal{O}(\mathrm{NMF})$ would have output earlier. We exclude this from the description for simplicity of notation. 
Whenever $\mathcal{O}(\mathrm{NMF})$ makes a query $q$, start a new auxiliary session of $\Pi$ with Alice, and run the code of $P_{2}$ honestly using input $q$. Since $\mathcal{O}(\mathrm{NMF})$ makes $t_{\mathrm{NMF}}$ different queries, in total, $t_{\mathrm{NMF}}$ auxiliary sessions of $\Pi$ are executed sequentially by Bob.

2. If $i<m \cdot n$, then on receiving the output $d_{i}$ from $\mathcal{O}(\mathrm{NMF})$ outputs, resume the suspended "main" session and send $d_{i}$ to Alice as the response to $a_{i}$. Otherwise, output the value $d_{i}=$ secret.

Lemma 5. Bob outputs the value secret in Experiment III with probability 1.

Completing the Proof of Theorem 4. The adversary $\mathcal{B}$ is simply the adversary Bob in Experiment III as described above. The proof of Theorem 4 then follows immediately from Lemma 4] and 5. We refer the reader to the full version for the missing proofs of the lemmas.

\section{References}

1. Agrawal, S., Goyal, V., Jain, A., Prabhakaran, M., Sahai, A.: New impossibility results for concurrent composition and a non-interactive completeness theorem for secure computation. In: Safavi-Naini, R. (ed.) CRYPTO 2012. LNCS, vol. 7417, pp. 443-460. Springer, Heidelberg (2012)

2. Barak, B., Canetti, R., Lindell, Y., Pass, R., Rabin, T.: Secure computation without authentication. In: Shoup, V. (ed.) CRYPTO 2005. LNCS, vol. 3621, pp. 361-377. Springer, Heidelberg (2005)

3. Barak, B., Canetti, R., Nielsen, J.B., Pass, R.: Universally composable protocols with relaxed set-up assumptions. In: FOCS, pp. 186-195 (2004)

4. Barak, B., Goldreich, O., Impagliazzo, R., Rudich, S., Sahai, A., Vadhan, S.P., Yang, K.: On the (im)possibility of obfuscating programs. In: Kilian, J. (ed.) CRYPTO 2001. LNCS, vol. 2139, pp. 1-18. Springer, Heidelberg (2001)

5. Barak, B., Prabhakaran, M., Sahai, A.: Concurrent non-malleable zero knowledge. In: FOCS, pp. 345-354 (2006)

6. Barak, B., Sahai, A.: How to play almost any mental game over the net - concurrent composition via super-polynomial simulation. In: FOCS, pp. 543-552 (2005)

7. Canetti, R.: Universally composable security: A new paradigm for cryptographic protocols. In: FOCS, pp. 136-147 (2001)

8. Canetti, R., Fischlin, M.: Universally composable commitments. In: Kilian, J. (ed.) CRYPTO 2001. LNCS, vol. 2139, pp. 19-40. Springer, Heidelberg (2001)

9. Canetti, R., Halevi, S., Katz, J., Lindell, Y., MacKenzie, P.: Universally composable password-based key exchange. In: Cramer, R. (ed.) EUROCRYPT 2005. LNCS, vol. 3494, pp. 404-421. Springer, Heidelberg (2005)

10. Canetti, R., Kushilevitz, E., Lindell, Y.: On the limitations of universally composable two-party computation without set-up assumptions. In: Biham, E. (ed.) EUROCRYPT 2003. LNCS, vol. 2656, pp. 68-86. Springer, Heidelberg (2003)

11. Canetti, R., Lin, H., Pass, R.: Adaptive hardness and composable security in the plain model from standard assumptions. In: FOCS, pp. 541-550 (2010)

12. Canetti, R., Lindell, Y., Ostrovsky, R., Sahai, A.: Universally composable two-party and multi-party secure computation. In: STOC, pp. 494-503 (2002) 
13. Canetti, R., Pass, R., Shelat, A.: Cryptography from sunspots: How to use an imperfect reference string. In: FOCS, pp. 249-259 (2007)

14. Dwork, C., Naor, M., Sahai, A.: Concurrent zero-knowledge. In: STOC (1998)

15. Garg, S., Goyal, V., Jain, A., Sahai, A.: Concurrently secure computation in constant rounds. In: Pointcheval, D., Johansson, T. (eds.) EUROCRYPT 2012. LNCS, vol. 7237, pp. 99-116. Springer, Heidelberg (2012)

16. Garg, S., Kumarasubramanian, A., Ostrovsky, R., Visconti, I.: Impossibility results for static input secure computation. In: Safavi-Naini, R. (ed.) CRYPTO 2012. LNCS, vol. 7417, pp. 424-442. Springer, Heidelberg (2012)

17. Gennaro, R., Lindell, Y.: A framework for password-based authenticated key exchange. In: Biham, E. (ed.) EUROCRYPT 2003. LNCS, vol. 2656, pp. 524-543. Springer, Heidelberg (2003)

18. Goldreich, O., Micali, S., Wigderson, A.: How to play ANY mental game. In: STOC, pp. 218-229 (1987)

19. Goldreich, O., Lindell, Y.: Session-key generation using human passwords only. In: Kilian, J. (ed.) CRYPTO 2001. LNCS, vol. 2139, pp. 408-432. Springer, Heidelberg (2001)

20. Goyal, V.: Positive results for concurrently secure computation in the plain model. In: FOCS (2012)

21. Goyal, V., Gupta, D., Jain, A.: What information is leaked under concurrent composition? Manuscript (2013)

22. Goyal, V., Ishai, Y., Sahai, A., Venkatesan, R., Wadia, A.: Founding cryptography on tamper-proof hardware tokens. In: Micciancio, D. (ed.) TCC 2010. LNCS, vol. 5978, pp. 308-326. Springer, Heidelberg (2010)

23. Goyal, V., Jain, A., Ostrovsky, R.: Password-authenticated session-key generation on the internet in the plain model. In: Rabin, T. (ed.) CRYPTO 2010. LNCS, vol. 6223, pp. 277-294. Springer, Heidelberg (2010)

24. Goyal, V., Sahai, A.: Resettably secure computation. In: Joux, A. (ed.) EUROCRYPT 2009. LNCS, vol. 5479, pp. 54-71. Springer, Heidelberg (2009)

25. Katz, J.: Universally composable multi-party computation using tamper-proof hardware. In: Naor, M. (ed.) EUROCRYPT 2007. LNCS, vol. 4515, pp. 115-128. Springer, Heidelberg (2007)

26. Katz, J., Ostrovsky, R., Yung, M.: Efficient password-authenticated key exchange using human-memorable passwords. In: Pfitzmann, B. (ed.) EUROCRYPT 2001. LNCS, vol. 2045, pp. 475-494. Springer, Heidelberg (2001)

27. Kidron, D., Lindell, Y.: Impossibility results for universal composability in publickey models and with fixed inputs. J. Cryptology 24(3), 517-544 (2011)

28. Kilian, J., Petrank, E.: Concurrent and resettable zero-knowledge in poly-logarithm rounds. In: STOC (2001)

29. Lin, H., Pass, R., Venkitasubramaniam, M.: A unified framework for concurrent security: universal composability from stand-alone non-malleability. In: STOC, pp. 179-188. ACM (2009)

30. Lindell, Y.: General composition and universal composability in secure multi-party computation. In: FOCS, pp. 394-403 (2003)

31. Lindell, Y.: Lower bounds for concurrent self composition. In: Naor, M. (ed.) TCC 2004. LNCS, vol. 2951, pp. 203-222. Springer, Heidelberg (2004)

32. Lindell, Y.: Lower bounds and impossibility results for concurrent self composition. J. Cryptology 21(2), 200-249 (2008)

33. Micali, S., Pass, R.: Local zero knowledge. In: STOC, pp. 306-315 (2006)

34. Micali, S., Pass, R., Rosen, A.: Input-indistinguishable computation. In: FOCS, pp. 367-378 (2006) 
35. Naor, M., Pinkas, B.: Oblivious transfer and polynomial evaluation. In: STOC (1999)

36. Naor, M., Pinkas, B.: Oblivious polynomial evaluation. SIAM J. Comput. (2006)

37. Pass, R.: Simulation in quasi-polynomial time, and its application to protocol composition. In: Biham, E. (ed.) EUROCRYPT 2003. LNCS, vol. 2656, pp. 160-176. Springer, Heidelberg (2003)

38. Pass, R.: Personal communication (2012)

39. Prabhakaran, M., Rosen, A., Sahai, A.: Concurrent zero knowledge with logarithmic round-complexity. In: FOCS, pp. 366-375 (2002)

40. Prabhakaran, M., Rosulek, M.: Cryptographic complexity of multi-party computation problems: Classifications and separations. In: Wagner, D. (ed.) CRYPTO 2008. LNCS, vol. 5157, pp. 262-279. Springer, Heidelberg (2008)

41. Prabhakaran, M., Sahai, A.: New notions of security: achieving universal composability without trusted setup. In: STOC, pp. 242-251 (2004)

42. Richardson, R., Kilian, J.: On the concurrent composition of zero-knowledge proofs. In: Stern, J. (ed.) EUROCRYPT 1999. LNCS, vol. 1592, pp. 415-431. Springer, Heidelberg (1999)

43. Chi-Chih Yao, A.: How to generate and exchange secrets. In: FOCS, pp. 162-167 (1986) 